\title{
Low Probability Cluster Head Selection Energy Efficient Routing in MANET using DEEC
}

\author{
Swati Shamkuwar \\ M-Tech Research Scholar, \\ Department of Computer Science \\ Engineering, KNP College of \\ Science \& Technology, Bhopal
}

\author{
Vimal Shukla, \\ Asst. Prof., Department of \\ Computer \\ Science Engineering \\ KNP College of Science \& \\ Technology, Bhopal
}

\author{
Navneet Manjhi \\ Asst. Prof., Department of \\ Computer \\ Science Engineering \\ KNP College of Science \& \\ Technology, Bhopal
}

\begin{abstract}
Mobile Ad-hoc Network is the prominent area of research to make wireless networking more robust and reliable for packet delivery among nodes of a small or big network through wireless channel. The routing protocols are also designed to keep in mind that the energy consumption of wireless nodes should be as minimum as possible. In MANET most of the devices are battery operated and saving energy increases the lifetime of the node to transfer information over network, in such case the routing protocol plays important role to increase the network lifetime. In this paper an efficient routing methodology is proposed which significantly increases the network lifetime. Here the distributed energy efficient clustering (DEEC) routing protocol is used with low probability cluster head selection approach, which enhances the network lifetime better than simple DEEC.
\end{abstract}

\section{Keywords}

DEEC Routing, Cluster Head(CH), Energy Efficient Routing, Low Probability Selection and MANET.

\section{INTRODUCTION}

Wireless Sensor Network (WSN) is an upcoming technology which has a wide range of application including infrastructure protection, industrial sensing and diagnostics, environment monitoring, context-aware computing (for example intelligent home and responsive environment). This network usually consists of a large number of nodes that bring themselves together to form a wireless network. The components of a WSN are sensor nodes, BS and monitored events (that is, an event that is required to be sensed in the environment) [7]. A usual sensor node is made of four building blocks: power unit, sensing unit, processing unit and communication unit [1]. The sensing component in a node measures certain physical characteristic like temperature or detects soil moisture of a location in which it is placed. The processing section is accountable for collection and processing captured data from its surrounding field. The wireless communication component of a sensor node is responsible for transmission or reception of captured data from one sensor node to another node or to an end user through the cluster head to the base station (BS). The sensor node, its processing and communication component requires energy to function as it has been expected, and the power component, which is of limited amount, is solely accountable for provision of energy to the three other components [1]. Based on application, the monitored event can either be dynamic or static in its operation. WSNs are typically deployed in an environment to monitor static or dynamic events. The measurement of static events (such a temperature, humidity) is very easy to carried out. On the other hand, dynamic events are typically noncooperative event is the movement of an unwanted vehicle in a battle field and the movement of whales in the ocean. They are not easy to monitor and they are not stable as they go up and down. Therefore, it is highly difficult to study energy saving schemes for sensing of dynamic event.

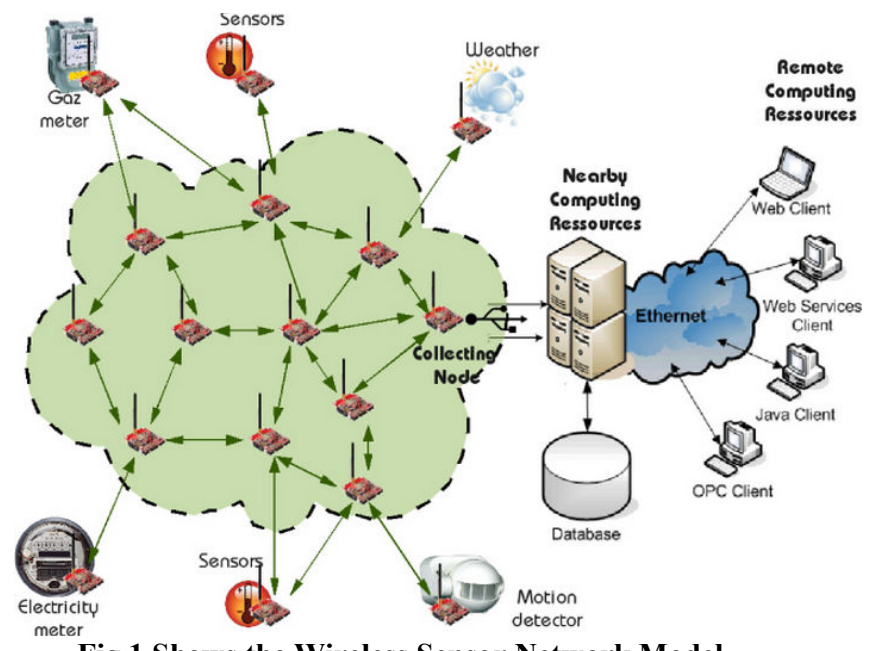

Fig.1 Shows the Wireless Sensor Network Model

Wireless sensor networks consist of small nodes with sensing, the computational and the wireless communications capabilities that can be deployed randomly or deterministically in an area from which the users wish to collect data. In general, wireless sensor networks contain hundreds or thousands of these sensor nodes that are generally identical. These sensor nodes have the ability to communicate either among each other or directly to abase station (BS). The network is highly distributed and the nodes are lightweight. A greater number of sensors will enable sensing over a larger area. As the developed of small, low-cost sensors become increasingly technically and economically practicable, a large number of these sensors could be networked to operate cooperatively unattended for a variety of applications. Such applications include military and civil applications like intrusion detection, target field imaging, inventory control and tactical surveillance. This section introduces the general components in a single node, its operating system and the wireless sensor network topology. 


\section{ROUTING TECHNIQUES}

Challenges encountered as a result of constrained energy supply and bandwidth in WSN when managing the network necessitates the need for development of energy awareness protocol at all levels of networking protocol stack. To present efficient power management in WSN, researches have been focus on areas such as system-level power awareness like radio communication hardware, low duty cycle work and energy-aware MAC protocol [7]. Also, it was observed that the network layer offers a better means through which reliable relaying of data and energy-efficient route setup within a network can help to maximize the network lifetime.

It should be noted that routing in WSN has much distinguishable features compare to contemporary communication and ad hoc networks [7]. These features are as follows:

- WSN cannot be built with global addressing (internet protocol address) scheme due to the enormous number of sensor nodes;

- There is significant redundancy in generated data because several sensors may gather the same data within a specified field. These redundancy bits need to be removed to increase the bandwidth utilization and also reduce energy consumption in the network;

- Transmission power of the system, processing capacity and storage are constraint factors to be considered when managing a WSN.

\section{Clustered Architecture:}

Hierarchical or cluster based routing methods are well known routing methods with a special advantage related to scalability and efficient communications. Hence, they are used for energy efficient routing in wireless sensor networks. In hierarchical routing, higher-energy nodes can be used to process and send the information, whereas low-energy nodes can be used to perform the sensing in the vicinity of the target. The creation of clusters and the assignment of special tasks to cluster heads contribute to the overall systems scalability. Nodes within a cluster lower the energy consumption by performing data aggregation and fusion, lowering the number of transmitted messages to the base station, thus prolonging network lifetime. Hierarchical routing is mainly comprised of two levels: one for the selection of cluster heads and the other for routing.

A clustered architecture consists of a cluster head, or Personal Area Network coordinator, which organizes sensor nodes, communicates for them to the BS and typically interfaces with another network. This structural design is well suited when data fusion is necessary. The cluster head fuses data gathered by member nodes and transmits the resulting information to the base station. A design of a clustered architecture is shown in Figure 2 In order for clustered networks for achieving the self-organization, the cluster formation and election process must be an autonomous, distributed procedure. This is achieved during network layer protocols, such as Low-Energy Adaptive Clustering Hierarchy (LEACH). [8, 9]

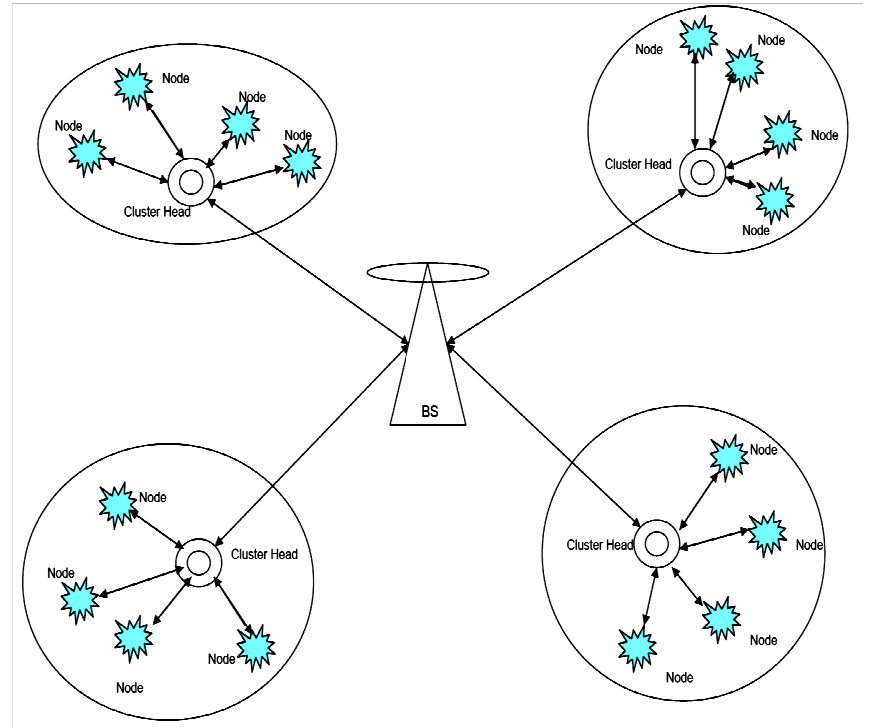

Fig.2 Clustered Architecture Illustrating Cluster Head Establishment[8]

A set of protocols for complete implementation of a layered architecture is described as a Unified Network Protocol Framework (UNPF). Three operations are integrated into the protocol structure of UNPF: network initialization and maintenance, Medium Access Control (MAC) and routing protocols. The BS broadcasts an identifying beacon on a common control channel. All nodes which receive the beacon broadcast the ir signal at their low power setting along with their own identification. Those nodes that the BS can directly communicate with form layer one. All nodes then transmit a beacon signal again. Nodes that receive this beacon again broadcast their signal at their low power setting along with their own identification. Therefore, the nodes of layer one establish layer- two nodes by recording the identification of the nodes with which they can communicate. The iterations continue until all nodes are identified with a layer. Thereafter, aperiodic beacon refreshes the architecture. [8]

\section{LEACH}

LEACH operates in two phases, setup and steady state. During the setup phase, LEACH minimizes energy dissipation by randomly selecting and periodically reselecting nodes as cluster heads. In this way, the high energy consumption experienced by cluster heads is distributed all through the network, so assuring that all cluster heads eventually expend equal energy. Later than selection, the cluster heads advertise their selection to all network nodes. The nodes are in turn associate themselves with the nearest cluster head based on the received signal strength of the selection advertisement. A TDMA schedule is then assigned for node communication. The steady-state phase is extensive long in comparison to the setup phase in order to minimize the overhead of cluster formation. Data transmission takes place during the steadystate phase based on the TDMA schedule established during setup. Energy is conserved by local processing and data aggregation at the cluster head. [9]

\section{Ad hoc On-demand Distance Vector:}

Ad hoc On-demand Distance Vector (AODV), as the name suggests is an on-demand protocol designed for mobile ad hoc networks [6]. This protocol responds quickly to changing link conditions and link breakages. The nodes mark the routes as invalid whenever there is a link breakage. AODV does not 
necessitate a node to maintain routes to destinations that are not in active communication. Loop freedom in AODV is ensured by using destination sequence numbers. These also allow nodes to use the most recent route to a target. The routing table information consist the destination address and the next hop address with the number of hops required to reach the destination. Also, the most recent destination sequence number associated with destination and lifetime of the route is stored in the table. If throughout the lifetime, the route is not used, the routing table entry is discarded.

\section{DEEC:}

In DEEC [3] protocol all nodes use the initial and residual energy level to define the cluster heads. DEEC estimate the ideal value of network lifetime to compute the reference energy that each node should expend during each round. In a two-level heterogeneous network, where we have two categories of nodes, $\mathrm{m}$. $\mathrm{N}$ advanced nodes with initial energy equal to $E o .(1+a)$ and $(1-m)$.N normal nodes, where the initial energy is equal to Eo. Where a and $\mathrm{m}$ are two variable which control the nodes percentage types (advanced or normal) and the total initial energy in the network $E_{\text {total }}$.

The value of Total Energy is given as

$$
E_{\text {total }}=N \cdot(1-m) \cdot E_{o}+N \cdot m \cdot E_{o} \cdot(1+a)(1)
$$

-The average energy of $r^{\text {th }}$ round is set as follows

$$
E(r)=\frac{1}{N} E_{\text {total }}(1-R)
$$

$\mathrm{R}$ represent the total rounds of the network lifetime and is defined as

$$
R=\frac{E_{\text {total }}}{E_{\text {Round }}}(3)
$$

- $E_{\text {Round }}$ is the total energy dissipated in the network during a round, is equal to: $E_{\text {Round }}=\mathrm{L}(2 \mathrm{NEelec}+\mathrm{NEDA}+$ $k E m p d^{4}$ toBS+NEfsd2toCH) (4)

$\mathrm{k}$ : number of clusters

EDA: data aggregation cost expended in the cluster heads

dto BS : average distance between the cluster head and the base station

dtoCH: average distance between the cluster members and the cluster head.

- Because it has been assumed that the nodes are uniformly distributed then get:

$$
\begin{gathered}
d t o C H=\frac{M}{\sqrt{2 k \pi}} \\
d t o B S=\frac{0.765 M}{2}
\end{gathered}
$$

\section{PROPOSED METHODOLOGY}

The wireless sensor network a subset of mobile ad-hoc network has lot of challenges to reduce the energy consumption of sensor nodes or wireless nodes to live longer in network and keep communicating with the network. Here we have to major areas by which a node can live longer and i.e. either make batteries (source of energy) equipped with nodes having larger in size or the material having larger charges saving capability but this approach having limited capabilities because the larger battery size make sensor node more bulk which is not feasible in any case, and to finding out the material has larger charge storing capability is also tough task to do.

Instead doing above things another method is to make transfer of information in network more efficient. For this many routing protocols has been given as we discussed in the previous sections. 


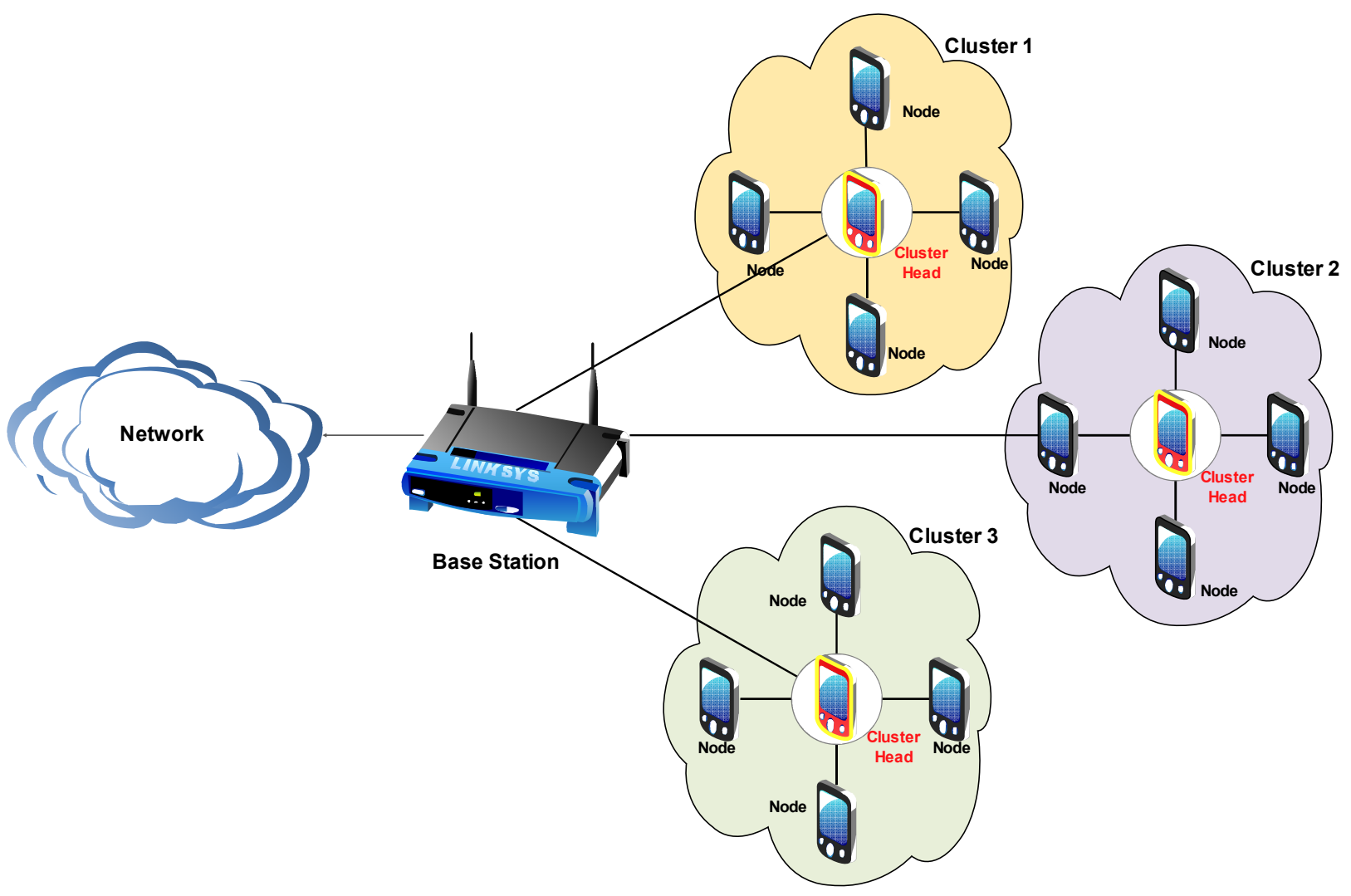

Fig. 3 Network Diagram of Proposed Methodology with DEEC Low Probability Cluster Head Selection Protocol

Here we are making changes in one of the routing protocol i.e. distributed energy efficient routing (DEEC), where changes are being made in the selection process of cluster heads. The proposed approach is to have the probability of cluster head selection is lower so that the cluster head do not change frequently and the energy can be conserve for such frequent changes in the network. The proposed diagram of network is presented in Fig.3

The flow of the proposed methodology is explained in the Fig. 3 In the proposed routing protocol the DEEC is modified a little bit and the protocol apply lower probability to select cluster heads which significantly reduce the consumption of energy. The proposed approach having following steps:
a) Start the program.
b) Initialization environmental variables
c) Generation of wireless sensor network model
d) Set loop for the number of rounds
e) Set number of alive nodes at the beginning of network
f) Check number of dead nodes
g) Select the cluster heads with defined probabilities (i.e. $2 \%$ as per proposed approach)
h) Calculate energy consumptions to transfer data between cluster head $(\mathrm{CH})$ to Base station(BS) and nodes $(\mathrm{N})$ to cluster head $(\mathrm{CH})$
i) Check alive nodes after data transfer
j) If alive nodes are $>0$ then Go back to Next Round (step c)
k) If alive nodes are $=0$ then calculate Throughput of the network
1) Compare and display results
m) End of program

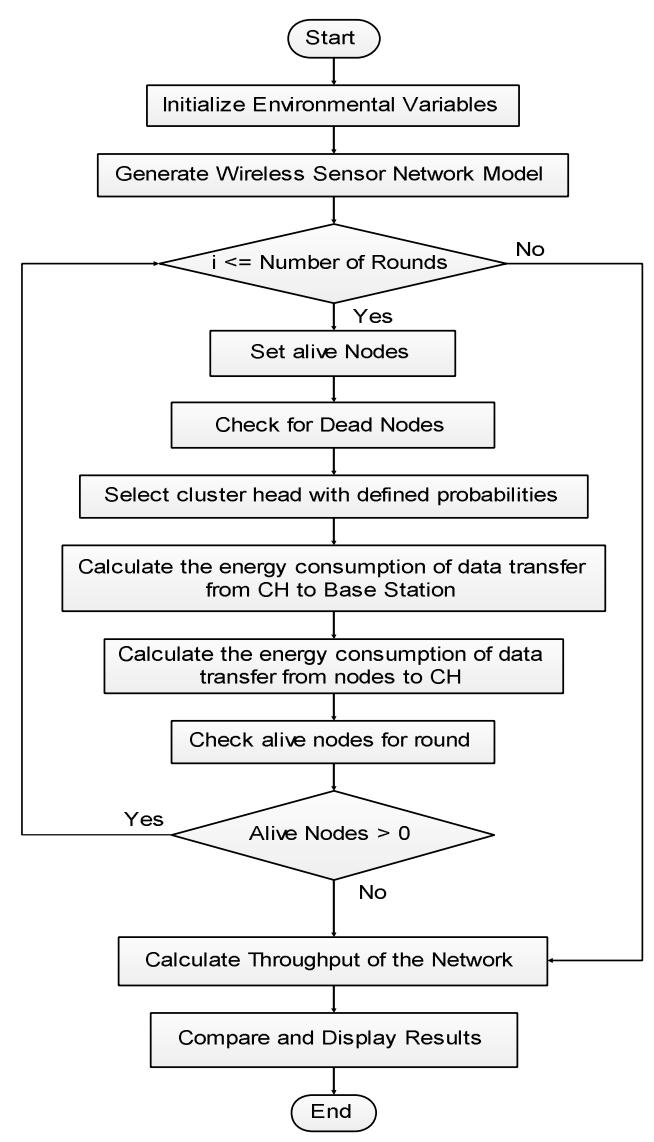

Fig. 4 Flow Chart of Proposed Methodology with DEEC Low Probability Cluster Head Selection Protocol 


\section{SIMULATION RESULTS}

Mobile Ad-hoc Network (MANET) is having lots of research areas to work on and here we have chosen routing protocol to make network lifetime little bit more than the previous one. The simulation performed on distributed energy efficient clustering(DEEC) which is based on choosing the clusters randomly with a head node which is a centre node through which all the communication being made to network.

The simulation of proposed methodology explained in the previous section is done in this section and the results of simulations are explained in below figures. In Fig. 4.1 the alive nodes contrast is shown during the network life and the comparison of various results of cluster head selection probability is made.

The results shown with the $2 \%, 10 \%$ (default), $25 \%$ and $50 \%$ cluster head selection probability. From the above results it is clear that if the cluster head selection is done with lower probability the network life is quite better than the default selection probability.

The green, red and blue graph has the lower network lifetime than black graph which represents for lower selection probability (i.e. $2 \%$ ).

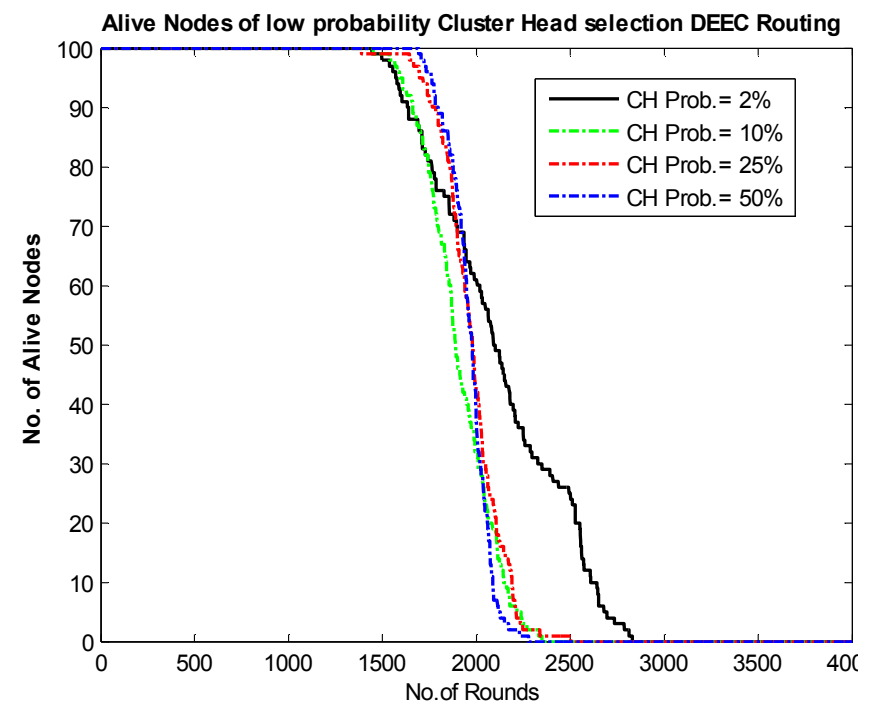

Fig. 5 Alive nodes of Low Probability Cluster Head Selection DEEC Routing

In Fig. 6 contrast of the dead nodes are shown and the difference is very clear that the with lower probability of cluster head selection the nodes started dying first but shortly with the network lifetime nodes are start dying with higher selection probability conditions and the lower probability selection system sustain longer among other probability conditions.

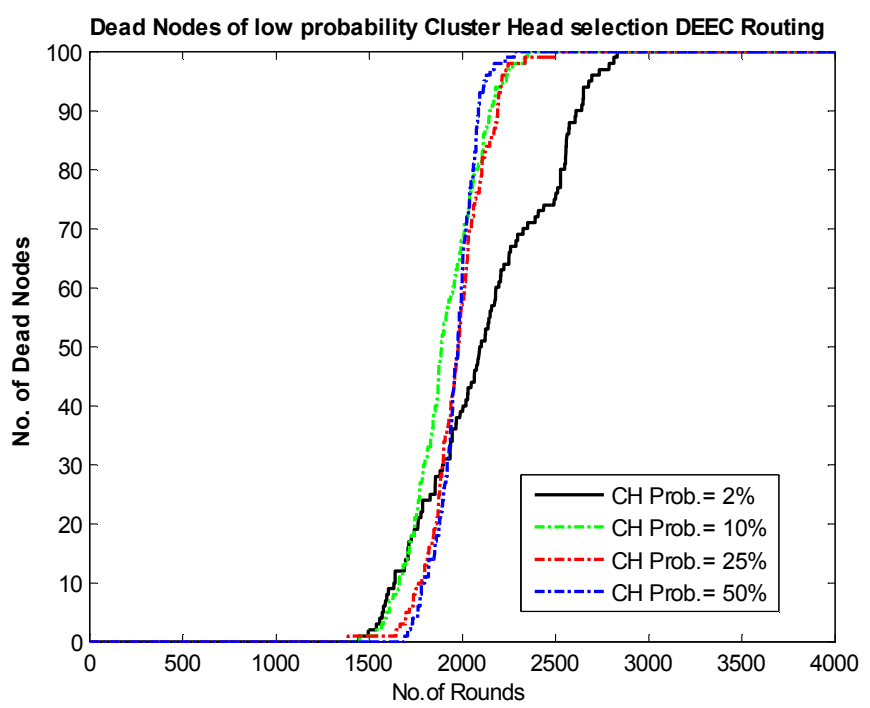

Fig. 6 Dead nodes of Low Probability Cluster Head Selection DEEC Routing

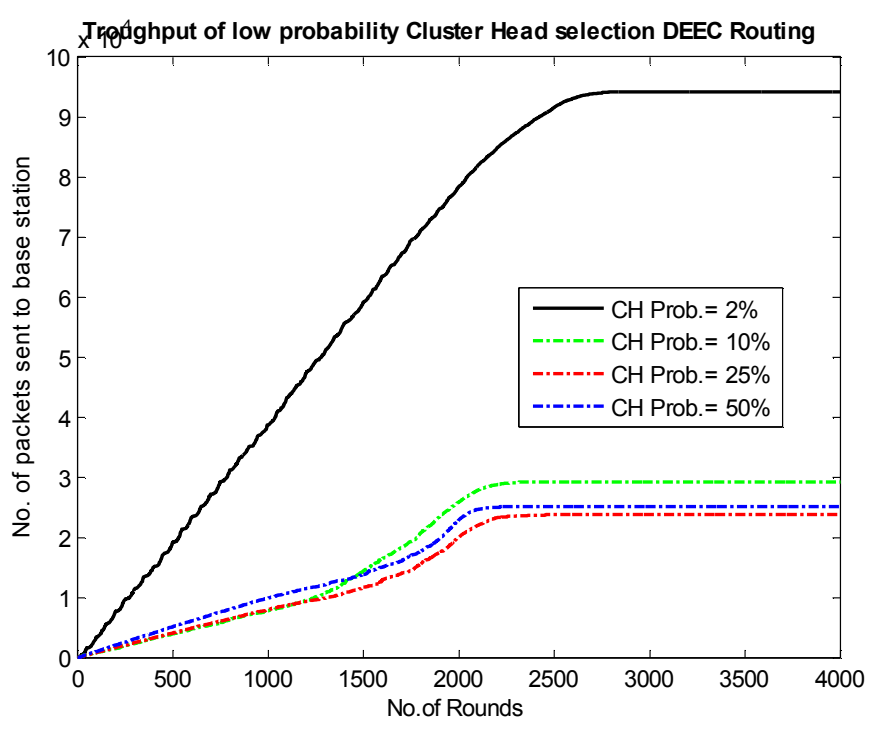

Fig. 7 Throughput of Low Probability Cluster Head Selection DEEC Routing

In Fig. 7 throughput of the proposed approach is compared with the higher and default probability systems. The throughputs of $10 \%, 25 \%$ and $50 \%$ cluster head selection probability are going constant after approximately 2200 rounds and for the $2 \%$ selection probability is will constant after 2700 rounds which is far better than higher probability conditions.

\section{CONCLUSION AND FUTURE SCOPE}

In the mobile ad-hoc network (MANET) there is need to be having longer life time to stay with the network, and the proposed methodology and its simulation results clearly declared that with the lower probability of cluster head selection the distributed energy efficient clustering (DEEC) routing can have the longer network lifetime better than higher probability selection schemes. With the analysis of other parameters like network area, initial energy etc. researcher will make out something more robust routing protocols which has lower energy consumption and higher network lifetime. 


\section{REFERENCES}

[1] Akyildiz, W. Su, Y. Sankarasubramaniam, E. Cayirci, survey on sensor networks, IEEE Communications Magazine, 40:8, 102-114, 2002.

[2] S.Mohanty and S.K.Patra, - A novel Bio-inspired Clustering algorithm for Wireless Sensor Networks,\| accepted in 3rd International Conference on Intelligent and Advanced Systems, Kuala Lumpur, Malaysia (ICIAS 2010).

[3] D. J. Cook and S.K. Das, Smart Environments: Technologies,Protocols, and Applications, John Wiley, New York, 2004.

[4] Tyagi S, Kumar N. A systematic review on clustering and routing techniques based upon LEACH protocol for wireless sensor networks, Journal of Network and Computer Applications, Elsevier, Vol. 36, issue 2, 2013, pp 623-645.

[5] C. Intanagonwiwat, R. Govindan, and D. Estrin, Directed diffusion: a scalable and robust communication paradigm for sensor networks, Proceedings of MobiCom'00, pp. 56-67, Boston, MA, USA, August 2000.

[6] W. R. Heinzelman, A. Chandrakasan, and H. Balakrishnan, Energyefficient communication protocol for wireless microsensor networks, Proceedings of the IEEE Hawaii International Conference on System Sciences, pp. 1-10, Maui, HI, USA, January 2000.

[7] W. Heinzelman, A. Chandrakasan, H. Balakrishnan, An application specific protocol architecture for wireless microsensor networks, IEEE Trans. Wireless communication, vol.1, no.4, pp 660-670, Oct. 2002.

[8] S. Lindsey and C. S. Raghavendra, PEGASIS: power efficient gathering in sensor information systems, Proceedings of the IEEE Aerospace Conference, Big Sky, MT, USA, pp 11251130, March 2002.

[9] Lindsey, S. Raghavendra, C. and Sivalingam, K, M. Data gathering in Sensor Networks using the energy delay metric, IEEE transactions on parallel and distributed systems, Vol. 13, No. 9, pp 924-935, 2002.

[10] A. Manjeshwar and D. P. Agrawal, TEEN: a protocol for enhanced efficiency in wireless sensor networks, Proceedings of the $1^{\text {st }}$ International Workshop on Parallel and Distributed Computing Issues in Wireless Networks and Mobile Computing, San Francisco, USA, April 2001.
[11] A. Manjeshwar and D. P. Agrawal, APTEEN: a hybrid protocol for efficient routing and comprehensive information retrieval in wireless sensor networks, Proceedings of the 2nd International Workshop on Parallel and Distributed Computing Issues in Wireless Networks and Mobile Computing, Ft. Lauderdale, FL, USA, April 2002.

[12] Bista, R., Yong-ki Kim, Jae-Woo Chang, A New Approach for Energy-Balanced Data Aggregation in Wireless Sensor Networks, Computer and Information Technology, 2009. CIT '09. Ninth IEEE International Conference on, vol.2, no., pp.9,15, 11-14 Oct. 2009.

[13] Yanwei Wu, Xiang-yang Li, Mo Li, Wei Lou, EnergyEfficient Wake-Up Scheduling for Data Collection and Aggregation, Parallel and Distributed Systems, IEEE Transactions on, vol.21, no.2, pp.275,287, Feb. 2010.

[14] Arabi, Z., HERF: A hybrid energy efficient routing using a fuzzy method in Wireless Sensor Networks, Intelligent and Advanced Systems (ICIAS), 2010 International Conference on , vol., no., pp.1,6, 15-17 June 2010.

[15] Katiyar, V., Chand, N., Gautam, G.C., Kumar, A., Improvement in LEACH protocol for large-scale wireless sensor networks, Emerging Trends in Electrical and Computer Technology (ICETECT), 2011 International Conference on, vol., no., pp.1070,1075, 23-24 March 2011.

[16] Chand, K.K., Bharati, P.V., Ramanjaneyulu, B.S., Optimized Energy Efficient Routing Protocol for life-time improvement in Wireless Sensor Networks, Advances in Engineering, Science and Management (ICAESM), 2012 International Conference on , vol., no., pp.345,349, 30-31 March 2012.

[17] Tyagi, S., Gupta, S.K., Tanwar, S., Kumar, N., EHE-LEACH : Enhanced heterogeneous LEACH protocol for lifetime enhancement of wireless SNs, Advances in Computing, Communications and Informatics (ICACCI), 2013 International Conference on, vol., no., pp.1485,1490, 22-25 Aug. 2013.

\section{AUTHOR'S PROFILE}

Swati Shamkuwar received the B.E. degree in 2011 inComputer Engineering from the Department of Computer Engineering of Maharashtra Academy of Engineering \& Educational Research, Alandi, Pune, affiliated to University of Pune. She is currently a master candidate in the Kailash Narayan Patidar College of Science \& Technology-Bhopal, India. Her research interests are energy efficient routing protocols in Wireless Sensor Networks. 